\title{
APPLICATION OF ARTIFICIAL NEURAL NETWORKS TO STRATIGRAPHIC CORRELATION
}

MALMGREN*, Björn A., Department of Marine Geology, Earth Sciences Centre, University of Göteborg, S-413 81 Göteborg, Sweden; NORDLUND, Ulf, Institute of Earth Sciences, University of Uppsala, Norbyvägen 22, S-752 36 Uppsala, Sweden.

Artificial neural networks, a branch of Artificial Intelligence, are computer systems formed by simple, highly interconnected processing elements that can learn a set of target vectors from an associated set of input signals. Neural networks learn by self-adjusting a set of parameters, using some pertinent algorithm to minimize the error between the desired output and network output. We have applied a neural network for solving a problem in chemostratigraphy, but the approach is equally well applicable to problems involving stratigraphic subdivision and correlation based on paleontological data.

The data used as an example are derived from four late Quaternary zones of volcanic ash of basaltic and rhyolithic origin from the Norwegian Sea. These ash layers span the oxygen isotope zones $1,5,7$, and 11 , respectively (last 420,000 years). The data consist of nine geochemical variables (oxides) determined in 183 samples.

We employed a 3-layer backpropagation (BP) neural network to determine how well it could learn to differentiate samples from the four ash zones on the basis of their geochemical composition, and thus how useful interzonal differences in the composition of these oxides would be for correlation of high-latitude North Atlantic strata. We created five random training and test sets in which the training sets contained $80 \%$ of the initial number of samples and the test sets the remaining $20 \%$. We employed a cross-validation (CV) technique to assess error rates of a neural network classifier on the test cases. We first determined the optimum number of neurons using one of the training-test set partitions as a test case. The minimum error rate was obtained for a network with 24 neurons in the hidden node layer. This configuration was then used to determine the overall $\mathrm{CV}$ error rates in the five training-test set partitions. Ten independent trials were run per partition. The BP network showed a CV error rate of $9.2 \%$ Hence, the network managed to classify on average $90.8 \%$ of the particles (33.6 out of 37 particles) in the test set with the correct ash zone and lava type and was thus highly successful.

Three statistical pattern recognition techniques, linear discriminant analysis, the $k$-nearest neighbor $(k$-NN) technique, and SIMCA (Soft Independent Modelling of Class Analogy) showed considerably higher $\mathrm{CV}$ error rates than the artificial neural networks, $38.4 \%, 30.8 \%$, and $28.7 \%$, respectively. This indicates that the BP network was indeed much more powerful than the statistical pattern-recognition techniques in correctly assigning the ash particles to the appropriate zone. 